\title{
The Quantum Condition That Should Have Been Assumed by Bohr When Deriving the Energy Levels of a Hydrogen Atom
}

\author{
Koshun Suto \\ Chudaiji Buddhist Temple, Isesaki, Japan \\ Email: koshun_suto129@mbr.nifty.com
}

How to cite this paper: Suto, K. (2021) The Quantum Condition That Should Have Been Assumed by Bohr When Deriving the Energy Levels of a Hydrogen Atom. Journal of Applied Mathematics and Physics, 9, 1230-1244.

https://doi.org/10.4236/jamp.2021.96084

Received: May 12, 2021

Accepted: June 13, 2021

Published: June 16, 2021

Copyright $\odot 2021$ by author(s) and Scientific Research Publishing Inc. This work is licensed under the Creative Commons Attribution International License (CC BY 4.0).

http://creativecommons.org/licenses/by/4.0/

\section{(c) (i) Open Access}

\begin{abstract}
Bohr assumed a quantum condition when deriving the energy levels of a hydrogen atom. This famous quantum condition was not derived logically, but it beautifully explained the energy levels of the hydrogen atom. Therefore, Bohr's quantum condition was accepted by physicists. However, the energy levels predicted by the eventually completed quantum mechanics do not match perfectly with the predictions of Bohr. For this reason, it cannot be said that Bohr's quantum condition is a perfectly correct assumption. Since the mass of an electron which moves inside a hydrogen atom varies, Bohr's quantum condition must be revised. However, the newly derived relativistic quantum condition is too complex to be assumed at the beginning. The velocity of an electron in a hydrogen atom is known as the Bohr velocity. This velocity can be derived from the formula for energy levels derived by Bohr. The velocity $v$ of an electron including the principal quantum number $n$ is given by $\alpha c / n$. This paper elucidates the fact that this formula is built into Bohr's quantum condition. It is also concluded in this paper that it is precisely this velocity formula that is the quantum condition that should have been assumed in the first place by Bohr. From Bohr's quantum condition, it is impossible to derive the relativistic energy levels of a hydrogen atom, but they can be derived from the new quantum condition. This paper proposes raising the status of the previously-known Bohr velocity formula.
\end{abstract}

\section{Keywords}

Relativistic Energy Levels of the Hydrogen Atom, Bohr's Quantum

Condition, Bohr Velocity, Einstein's Energy-Momentum Relationship, Suto's Energy-Momentum Relationship, Relativistic Kinetic Energy 


\section{Introduction}

$\mathrm{N}$. Bohr was the first to derive the energy levels of an electron forming a hydrogen atom (this will be abbreviated below as energy levels of the hydrogen atom). This Introduction reviews the history up to derivation of the energy levels of the hydrogen atom with the assistance of the writings of Dr. H. Ezawa in Japanese.

In 1884, J. J. Balmer noticed that the wavelengths $\lambda$ of the spectral lines emitted from a hydrogen atom could be described with the following formula.

$$
\lambda=B \frac{n^{2}}{n^{2}-4}, \quad B=3.6456 \times 10^{-7} \mathrm{~m} .
$$

After that, W. Ritz transformed this formula as follows.

$$
\frac{1}{\lambda}=\frac{4}{B}\left(\frac{1}{2^{2}}-\frac{1}{n^{2}}\right), \quad n=3,4, \cdots
$$

Ritz also generalized Formula (2) as follows.

$$
\frac{1}{\lambda}=R\left(\frac{1}{m^{2}}-\frac{1}{n^{2}}\right), \quad n=m+1, m+2, \cdots ; m=1,2, \cdots .
$$

Bohr tried multiplying both sides of Formula (3) by $h c$. When this is done,

$$
h \frac{c}{\lambda}=h v=\frac{h c R}{m^{2}}-\frac{h c R}{n^{2}}, \quad m<n .
$$

From Formula (4), Bohr predicted the following relationship.

$$
h v=-E_{m}+E_{n} .
$$

The energy of the hydrogen atom is discontinuous. Bohr thought that when the electron transitions from a state with energy $E_{n}$ to a state with energy $E_{m}$, the electron emits a photon with energy $h v$. He also obtained the following formula for energy levels.

$$
E_{n}=-\frac{h c R}{n^{2}} .
$$

At the time, the value of $c R$ was known through experiment, but details concerning $R$ were not known. Thus Bohr decided to derive the energy levels of the hydrogen atom using another method.

First, Bohr considered the case where the electron moves at constant speed around the atomic nucleus (proton). If $r$ is taken to be the radius of a circular orbit, and $v$ is taken to be the speed of the electron, then the following Newtonian equation of motion holds.

$$
\frac{m_{\mathrm{e}} v^{2}}{r}=\frac{1}{4 \pi \varepsilon_{0}} \frac{e^{2}}{r^{2}}
$$

This equation indicates the equality of the centrifugal force acting on the electron (left side) and the Coulomb attraction received by the electron from the atomic nucleus. Here, the electron mass was set to $m_{\mathrm{e}}$, and the charge was set to $-e$.

Also, since the energy of the electron can be expressed by the sum of the ki- 
netic energy $K$ and potential energy $V(r)$,

$$
E=\frac{m_{\mathrm{e}} v^{2}}{2}-\frac{1}{4 \pi \varepsilon_{0}} \frac{e^{2}}{r} .
$$

According to the Virial theorem, $2 K=-V(r)$ in the case of a circular orbit, and thus the energy can be written as follows (the discussion here concerns a circular orbit as a special form of an elliptical orbit).

$$
\begin{gathered}
E=-\frac{m_{\mathrm{e}} v^{2}}{2} . \\
E=-K=-\frac{1}{2} \frac{1}{4 \pi \varepsilon_{0}} \frac{e^{2}}{r} .
\end{gathered}
$$

Here, if both sides of Formula (10) are squared,

$$
E^{2}=\frac{1}{4}\left(\frac{1}{4 \pi \varepsilon_{0}}\right)^{2} \frac{e^{4}}{r^{2}}
$$

Next, the following equation is obtained by dividing Formula (11) by Formula (9).

$$
E=-\frac{2}{m_{\mathrm{e}} v^{2}} \cdot \frac{1}{4}\left(\frac{1}{4 \pi \varepsilon_{0}}\right)^{2} \frac{e^{4}}{r^{2}}=-\frac{m_{\mathrm{e}}}{2}\left(\frac{1}{4 \pi \varepsilon_{0}}\right)^{2} \frac{e^{4}}{\left(m_{\mathrm{e}} v r\right)^{2}} .
$$

Incidentally, the angular momentum $L$ when an electron moves in a circle can be expressed as mvr. Here, if the number $n$ is affixed to the energy $E$ and angular momentum $L$, then Formula (12) becomes as follows.

$$
E_{n}=-\frac{m_{\mathrm{e}}}{2}\left(\frac{e^{2}}{4 \pi \varepsilon_{0}}\right)^{2} \frac{1}{L_{n}^{2}} .
$$

The energy in Formula (6) could be found through calculation. Bohr believed Formulas (6) and (13) to be equal. Thus,

$$
\frac{h c R}{n^{2}}=\frac{m_{\mathrm{e}}}{2}\left(\frac{e^{2}}{4 \pi \varepsilon_{0}}\right)^{2} \frac{1}{L_{n}^{2}} .
$$

It was found that $L$, for which a unique value was not known, could be expressed with the following equation.

$$
L_{n}=\frac{e^{2}}{4 \pi \varepsilon_{0}}\left(\frac{m_{\mathrm{e}}}{2 h c R}\right)^{1 / 2} \cdot n .
$$

Bohr substituted in the not very precise numeric values for physical quantities that were known at the time and conjectured $L_{n}$ to be as follows.

$$
L_{n}=\frac{h}{2 \pi} n=n \hbar, \quad n=1,2, \cdots .
$$

If Formula (16) is assumed, then Formula (13) becomes as follows [1].

$$
E_{\mathrm{BO}, n}=-\frac{1}{2}\left(\frac{1}{4 \pi \varepsilon_{0}}\right)^{2} \frac{m_{\mathrm{e}} e^{4}}{\hbar^{2}} \cdot \frac{1}{n^{2}}
$$




$$
\begin{aligned}
& =-\frac{m_{\mathrm{e}} c^{2}}{2}\left(\frac{e^{2}}{4 \pi \varepsilon_{0} \hbar c}\right)^{2} \frac{1}{n^{2}} \\
& =-\frac{\alpha^{2} m_{\mathrm{e}} c^{2}}{2 n^{2}} .
\end{aligned}
$$

Here, $E_{\mathrm{BO}, n}$ signifies the energy levels derived by Bohr. Also, $\alpha$ is the following fine-structure constant.

$$
\alpha=\frac{e^{2}}{4 \pi \varepsilon_{0} \hbar c}=7.2973525693 \times 10^{-3} .
$$

Formula (17) is not a logically derived formula. It is a formula derived by assuming the quantum condition in Formula (16).

At the time, L. de Broglie noticed that light, thought to be a wave in the classical theory, exhibits particle characteristics. He also predicted that the electron, thought to be a particle, would exhibit wave characteristics. He also assumed that when the wavelength $\lambda$ of the wave accompanying an electron in circular motion satisfies the following relationship, that electron is state.

$$
\frac{2 \pi r}{\lambda}=n, \quad n=1,2, \cdots
$$

The following relationship holds between the momentum $p$ and wavelength $\lambda$ of the electron.

$$
\lambda=\frac{h}{p} .
$$

Substituting the $\lambda$ of Formula (20) into Formula (19) and also taking into account Formula (16), the following formula can be derived.

$$
2 \pi r p_{n}=2 \pi L_{n}=n h, \quad n=1,2, \cdots .
$$

According to de Broglie, Bohr's quantum condition was able to acquire a substantive meaning, and thus it came to be that the energy levels of the hydrogen atom in Formula (17), found by assuming Formula (16), were believed to be correct.

Also, if $E_{n}$ in Formula (17) is substituted into Formula (10), then the following formula can be derived as the orbital radius of the electron.

$$
r_{\mathrm{BO}, n}=4 \pi \varepsilon_{0} \frac{\hbar^{2}}{m_{\mathrm{e}} e^{2}} n^{2}=a_{\mathrm{B}} n^{2}, \quad n=1,2, \cdots .
$$

Here, $r_{\mathrm{BO}, n}$ is the orbital radius of the electron predicted by Bohr's theory. Also, $a_{\mathrm{B}}$ is the orbital radius when $n=1$, i.e., the Bohr radius. The content of this paper thus far simply reiterates the information in another paper by the author [2]. However, this was deemed necessary for the discussion in subsequent sections. The content of this introduction is a shared understanding of physicists.

\section{Discussion of Bohr's Quantum Condition}

Bohr's quantum condition was not logically derived. Bohr's quantum condition 
was accepted because it enabled the energy levels (17a) of a hydrogen atom to be derived correctly. However, the value of Formula (17a) does not match perfectly with the value predicted by the completed theory of quantum mechanics.

This is likely because Bohr did not take account of the theory of relativity. When the theory of relativity is taken into account, Bohr's quantum condition (21) and de Broglie's hypothesis (19) must be revised.

\subsection{Relationship Enfolded in Bohr's Quantum Condition}

Bohr thought the following quantum condition was necessary to find the energy levels of the hydrogen atom.

$$
m_{\mathrm{e}} v_{n} \cdot 2 \pi r_{\mathrm{BO}, n}=2 \pi n \hbar .
$$

In Bohr's theory, the energy levels of the hydrogen atom are treated non-relativistically, and thus here the momentum of the electron is taken to be $m_{\mathrm{e}} v$. Also, the Planck constant $h$ can be written as follows [3].

$$
\hbar=\frac{h}{2 \pi}=\frac{m_{\mathrm{e}} c \lambda_{\mathrm{C}}}{2 \pi} .
$$

$\lambda_{\mathrm{C}}$ is the Compton wavelength of the electron.

When Formula (24) is used, the fine-structure constant $\alpha$ can be expressed as follows.

$$
\alpha=\frac{e^{2}}{4 \pi \varepsilon_{0} \hbar c}=\frac{e^{2}}{2 \varepsilon_{0} m_{\mathrm{e}} c^{2} \lambda_{\mathrm{C}}} .
$$

Also, the classical electron radius $r_{\mathrm{e}}$ is defined as follows.

$$
r_{\mathrm{e}}=\frac{e^{2}}{4 \pi \varepsilon_{0} m_{\mathrm{e}} c^{2}} .
$$

If $r_{\mathrm{e}} / \alpha$ is calculated here,

$$
\frac{r_{\mathrm{e}}}{\alpha}=\frac{\lambda_{\mathrm{C}}}{2 \pi} .
$$

If Formula (22) is written using $r_{\mathrm{e}}$ and $\alpha$, the result is as follows.

$$
r_{\mathrm{BO}, n}=4 \pi \varepsilon_{0} \frac{\hbar^{2}}{m_{\mathrm{e}} e^{2}} n^{2}=\frac{e^{2}}{4 \pi \varepsilon_{0} m_{\mathrm{e}} c^{2}}\left(\frac{4 \pi \varepsilon_{0} \hbar c}{e^{2}}\right)^{2} n^{2}=\frac{r_{\mathrm{e}}}{\alpha^{2}} n^{2} .
$$

Next, if $\hbar$ in Formula (24) and $r_{\mathrm{BO}, n}$ in Formula (28) are substituted into Formula (23),

$$
m_{\mathrm{e}} v_{n} \cdot 2 \pi \frac{r_{\mathrm{e}}}{\alpha^{2}} n^{2}=2 \pi n \frac{m_{\mathrm{e}} c \lambda_{\mathrm{C}}}{2 \pi} .
$$

If Formula (27) is also used, then Formula (29) can be written as follows.

$$
m_{\mathrm{e}} v_{n} \cdot 2 \pi \frac{r_{\mathrm{e}}}{\alpha^{2}} n^{2}=2 \pi n \frac{m_{\mathrm{e}} c r_{\mathrm{e}}}{\alpha} .
$$

From this, the following relationship can be derived.

$$
\frac{v_{n}}{c}=\frac{\alpha}{n} .
$$


Due to Formula (31), it is possible to identify discontinuous states that are permissible in terms of quantum mechanics in the continuous motions of classical theory.

\subsection{Various Formulas Derivable from Formula (31)}

\subsubsection{Bohr's Quantum Condition}

It was possible to derive Formula (31) from Bohr's quantum condition (23), and thus it should be possible to derive Formula (23) from Formula (31). First, both sides of Formula (31) are multiplied by $m_{\mathrm{e}} \cdot 2 \pi r_{\mathrm{BO}, n}$. Next, when the value of Formula (22) is substituted for $r_{\mathrm{BO}, n}$ on the right side,

$$
m_{\mathrm{e}} v_{n} \cdot 2 \pi r_{\mathrm{BO}, n}=\frac{m_{\mathrm{e}} c}{n}\left(\frac{e^{2}}{4 \pi \varepsilon_{0} \hbar c} \cdot 2 \pi \cdot 4 \pi \varepsilon_{0} \frac{\hbar^{2}}{m_{\mathrm{e}} e^{2}} n^{2}=2 \pi n \hbar .\right.
$$

With this, it was possible to derive Bohr's quantum condition (23) from Formula (31).

This shows that the electron mass which appears in Bohr's quantum condition is rest mass.

\subsubsection{Bohr's Energy Levels (17)}

When both sides of Formula (31) are squared, and then multiplied by $m_{\mathrm{e}} / 2$,

$$
\frac{1}{2} \frac{m_{\mathrm{e}} v_{n}^{2}}{c^{2}}=\frac{1}{2} \frac{m_{\mathrm{e}} \alpha^{2}}{n^{2}}
$$

Hence,

$$
E_{\mathrm{BO}, n}=-\frac{1}{2} m_{\mathrm{e}} v_{n}^{2}=-\frac{\alpha^{2} m_{\mathrm{e}} c^{2}}{2 n^{2}}
$$

If Formula (31) is taken as a departure point, the energy levels of the hydrogen atom derived by Bohr can be derived immediately. Formula (31) has tremendous power. However, from a relativistic perspective, $(1 / 2) m_{\mathrm{e}} v_{n}^{2}$ is an approximation of the kinetic energy of the electron. Therefore, the energy in Formula (34) is also an approximation of the true value.

\section{The Relation between Kinetic Energy and Momentum Derived from the STR Relationship}

The energy-momentum relationship in the special theory of relativity (STR) holds in an isolated system in free space. Here, if $m_{0} c^{2}$ is the rest mass energy and $m c^{2}$ is the relativistic energy, the relationship can be written as follows.

$$
\left(m_{0} c^{2}\right)^{2}+p^{2} c^{2}=\left(m c^{2}\right)^{2} \text {. }
$$

First, it is clear that the following formula holds.

$$
\left[m_{0} c^{2}+\left(m c^{2}-m_{0} c^{2}\right)\right]^{2}=\left(m c^{2}\right)^{2} .
$$

Expanding the left side of this equation yields the following.

$$
m_{0}^{2} c^{4}+\left(m^{2} c^{4}-m_{0}^{2} c^{4}\right)=\left(m_{0} c^{2}\right)^{2}+\left(m+m_{0}\right)\left(m c^{2}-m_{0} c^{2}\right) c^{2} .
$$


Using this, Formula (36) becomes as follows.

$$
\left(m_{0} c^{2}\right)^{2}+\left(m+m_{0}\right)\left(m c^{2}-m_{0} c^{2}\right) c^{2}=\left(m c^{2}\right)^{2} .
$$

Incidentally, Sommerfeld and Einstein defined relativistic kinetic energy as follows [4].

$$
K_{\mathrm{re}}=m c^{2}-m_{0} c^{2} .
$$

Since this equation and Formula (35) are equal, the following relationship must hold when Formula (39) is taken into account.

$$
p^{2}=\left(m_{0}+m\right)\left(m c^{2}-m_{0} c^{2}\right)=\left(m_{0}+m\right) K_{\mathrm{re}} .
$$

The following formula is obtained from this.

$$
K_{\mathrm{re}}=\frac{p^{2}}{m+m_{0}},
$$

Formula (41) is the formula for relativistic kinetic energy [2]. Classical (nonrelativistic) kinetic energy, in contrast, is defined as follows.

$$
K_{\mathrm{cl}}=\frac{1}{2} m_{0} v^{2}=\frac{p^{2}}{2 m_{0}} .
$$

Formula (41) describes the relativistic kinetic energy of an electron in a hydrogen atom, and Formula (42) describes the classical kinetic energy of an electron.

Next, the relativistic kinetic energy of an electron in a hydrogen atom is defined as follows by referring to Formula (41).

$$
K_{\mathrm{re}, n}=\frac{p_{\mathrm{re}, n}^{2}}{m_{\mathrm{e}}+m_{n}}, \quad p_{\mathrm{re}, n}=m_{n} v_{n} .
$$

Here, $m_{n}$ is the relativistic mass of the electron. Also, $p_{\mathrm{re}, n}$ indicates the relativistic momentum of the electron.

Incidentally, the energy of an electron at rest in an isolated system in free space is $m_{\mathrm{e}} c^{2}$. Here, we consider the case where this electron is drawn in by the electrical attraction of the proton, and forms a hydrogen atom. At this time, the electron emits a photon to the outside. Therefore, the relativistic energy of an electron in a hydrogen atom $m_{n} c^{2}$ becomes smaller than the rest mass energy $m_{\mathrm{e}} c^{2}$. That is,

$$
m_{n} c^{2}<m_{\mathrm{e}} c^{2}
$$

The behavior of an electron inside an atom, where there is potential energy, cannot be described with the relationship of Einstein (35). Caution is necessary because it is completely overlooked in Formula (44).

Now, referring to Formula (39), it is natural to define the relativistic kinetic energy of an electron in a hydrogen atom as follows.

$$
K_{\mathrm{re}, n}=-E_{\mathrm{re}, n}=m_{\mathrm{e}} c^{2}-m_{n} c^{2} .
$$

This paper defines $E_{\mathrm{re}, n}$ as the relativistic energy levels of the hydrogen atom 
derived at the level of classical quantum theory. (The quantum number used here is just the principal quantum number. Therefore, $E_{\mathrm{re}, n}$ is not a formula that predicts all the relativistic energy levels of the hydrogen atom.)

However, the term "relativistic" used here does not mean based on the STR. It means that the expression takes into account the fact that the mass of the electron varies due to velocity. According to the STR, the electron's mass increases when its velocity increases. However, inside the hydrogen atom, the mass of the electron decreases when the velocity of the electron increases. Attention must be paid to the fact that, inside the hydrogen atom, the relativistic mass of the electron $m_{n}$ is smaller than the rest mass $m_{\mathrm{e}}$.

In this way, two formulas have been obtained for the relativistic kinetic energy of the electron in a hydrogen atom (Formulas (43), and (45)).

The following Figure 1 illustrates the energy levels $E_{\mathrm{BO}, n}$ derived by Bohr and the energy levels $E_{\mathrm{re}, n}$ derived in this paper.

Incidentally, the following equation can be derived from Formulas (43) and (45).

$$
\frac{p_{\mathrm{re}, n}^{2}}{m_{\mathrm{e}}+m_{n}}=m_{\mathrm{e}} c^{2}-m_{n} c^{2} .
$$

Rearranging this, the following relationship can be derived.

$$
\left(m_{n} c^{2}\right)^{2}+p_{\mathrm{re}, n}^{2} c^{2}=\left(m_{\mathrm{e}} c^{2}\right)^{2} .
$$

Formula (47) is the energy-momentum relationship applicable to the electron in a hydrogen atom (the author calls this "Suto's energy-momentum relationship").

The author already derived this relationship (47) using another method [5] [6]. The difference between Einstein's relationships (35) and (47) arises due to the presence/absence of potential energy.

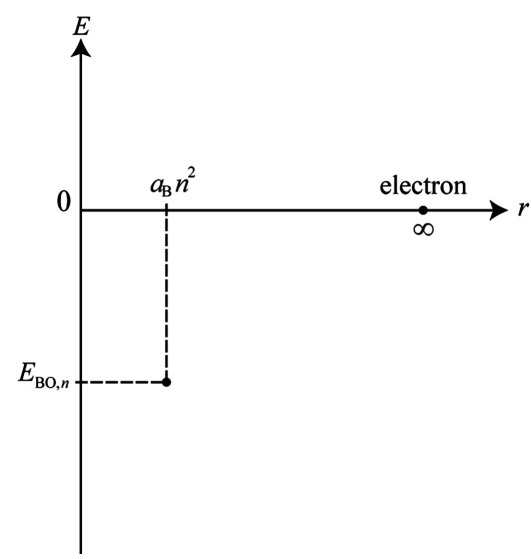

(a)

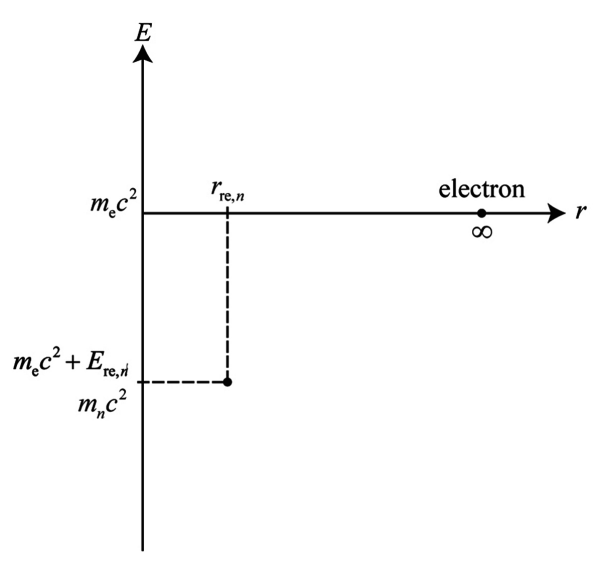

(b)

Figure 1. (a) Bohr's theory asserts that the energy of an electron at rest at the position $r=\infty$ is zero; (b) In this paper, $m_{\mathrm{e}} c^{2}$ is taken to be the energy of an electron at rest at the position $r=\infty$. The energy levels in this paper were derived from an absolute scale which takes into account the special theory of relativity. 
Now, if the definition of momentum for Formula (43) is taken into account, Formula (47) can be written as follows.

$$
\left(m_{n} c^{2}\right)^{2}+\left(m_{n} v_{n}\right)^{2} c^{2}=\left(m_{\mathrm{e}} c^{2}\right)^{2} .
$$

Using Formula (31) here, Formulas (48) can be written as follows.

$$
\left(m_{n} c^{2}\right)^{2}+m_{n}^{2}\left(\frac{\alpha c}{n}\right)^{2} c^{2}=\left(m_{\mathrm{e}} c^{2}\right)^{2} .
$$

The relation between $m_{n}$ and $m_{\mathrm{e}}$ is as follows due to Formula (49).

$$
\frac{m_{\mathrm{e}}}{m_{n}}=\left(1+\frac{\alpha^{2}}{n^{2}}\right)^{1 / 2} \text {. }
$$

According to the predictions of the STR, the mass of a moving object is greater than when it is at rest. However, in the case of an electron in a hydrogen atom, that principle is reversed, and the mass of the moving electron is smaller than the rest mass of the electron.

Now, if Formula (47) is solved for $p_{\text {re, } n}$,

$$
p_{\text {re }, n}=c\left(m_{\mathrm{e}}^{2}-m_{n}^{2}\right)^{1 / 2} \text {. }
$$

If Formula (50) is also taken into consideration,

$$
p_{\mathrm{re}, n}=m_{\mathrm{e}} c\left(\frac{\alpha^{2}}{n^{2}+\alpha^{2}}\right)^{1 / 2} \text {. }
$$

Incidentally, the following equation holds due to Formulas (10) and (45).

$$
\frac{1}{2} \frac{1}{4 \pi \varepsilon_{0}} \frac{e^{2}}{r_{\mathrm{re}, n}}=m_{\mathrm{e}} c^{2}-m_{n} c^{2} .
$$

Finding $r_{\text {re,n }}$ from Formula (53),

$$
r_{\mathrm{re}, n}=\frac{r_{\mathrm{e}}}{2} \frac{m_{\mathrm{e}}}{m_{\mathrm{e}}-m_{n}} .
$$

Next, if we calculate the denominator of Formula (54),

$$
\frac{m_{\mathrm{e}}}{m_{\mathrm{e}}-m_{n}}=\frac{1}{1-\frac{1}{\left(1-\alpha^{2} / n^{2}\right)^{1 / 2}}}=\frac{\left(n^{2}+\alpha^{2}\right)^{1 / 2}}{\left(n^{2}+\alpha^{2}\right)^{1 / 2}-n} .
$$

From this, $r_{\mathrm{re}, n}$ becomes as follows

$$
r_{\mathrm{re}, n}=\frac{r_{\mathrm{e}}}{2} \cdot \frac{\left(n^{2}+\alpha^{2}\right)^{1 / 2}}{\left(n^{2}+\alpha^{2}\right)^{1 / 2}-n} .
$$

Also, rewriting Formula (56), it becomes as follows.

$$
r_{\mathrm{re}, n}=\frac{r_{\mathrm{e}}}{2}\left[1+\frac{n}{\left(n^{2}+\alpha^{2}\right)^{1 / 2}-n}\right] \text {. }
$$




\section{Bohr's Quantum Condition and De Broglie's Hypothesis Derived from a Relativistic Standpoint}

In the discussion in the previous section, it was possible to find $p_{\text {re, } n}$ and $r_{\text {re, } n}$ for relativistically deriving Bohr's quantum condition (23) and de Broglie's hypothesis (19).

\subsection{Bohr's Quantum Condition}

If the values of Formulas (52) and (56) are substituted for $p_{\mathrm{re}, n}$ and $r_{\mathrm{re}, n}$ in Bohr's quantum condition (23), then the left side of Formula (23) becomes as follows.

$$
\begin{aligned}
p_{\text {re }, n} \cdot 2 \pi r_{\text {re }, n} & =m_{\mathrm{e}} c\left(\frac{\alpha^{2}}{n^{2}+\alpha^{2}}\right)^{1 / 2} \cdot 2 \pi \frac{r_{\mathrm{e}}}{2} \frac{\left(n^{2}+\alpha^{2}\right)^{1 / 2}}{\left(n^{2}+\alpha^{2}\right)^{1 / 2}-n} \\
& =m_{\mathrm{e}} c \alpha \cdot 2 \pi \frac{r_{\mathrm{e}}}{2} \frac{1}{\left(n^{2}+\alpha^{2}\right)^{1 / 2}-n} \\
& =m_{\mathrm{e}} c \alpha \cdot 2 \pi \frac{r_{\mathrm{e}}}{2 \alpha^{2}} \cdot n\left[\left(1+\frac{\alpha^{2}}{n^{2}}\right)^{1 / 2}+1\right]
\end{aligned}
$$

Here, we use the fact that Formula (27) can be written as follows.

$$
\frac{r_{\mathrm{e}}}{\alpha}=\frac{\lambda_{\mathrm{C}}}{2 \pi}=\frac{\hbar}{m_{\mathrm{e}} c} .
$$

When this is done, Formula (58) becomes as follows.

$$
p_{\mathrm{re}, n} \cdot 2 \pi r_{\mathrm{re}, n}=m_{\mathrm{e}} c \cdot 2 \pi \frac{r_{\mathrm{e}}}{2 \alpha} \cdot n\left[1+\left(1+\frac{\alpha^{2}}{n^{2}}\right)^{1 / 2}\right]=2 \pi \frac{n \hbar}{2}\left[1+\left(1+\frac{\alpha^{2}}{n^{2}}\right)^{1 / 2}\right] \text {. }
$$

In this paper, the following equation is called the relativistic quantum condition.

$$
p_{\mathrm{re}, n} \cdot 2 \pi r_{\mathrm{re}, n}=2 \pi \frac{n \hbar}{2}\left[1+\left(1+\frac{\alpha^{2}}{n^{2}}\right)^{1 / 2}\right]
$$

Next, if the part in parentheses on the right side of Formula (60) is developed as a Taylor expansion, the result is as follows.

$$
\left(1+\frac{\alpha^{2}}{n^{2}}\right)^{1 / 2} \approx 1+\frac{\alpha^{2}}{2 n^{2}}-\frac{\alpha^{4}}{8 n^{4}}+\frac{\alpha^{6}}{16 n^{6}}
$$

Thus,

$$
1+\left(1+\frac{\alpha^{2}}{n^{2}}\right)^{1 / 2} \approx 2+\frac{\alpha^{2}}{2 n^{2}}
$$

Here, $\alpha^{2}=5.325 \times 10^{-4}$, and thus the left side of Formula (62) is approximate,

$$
1+\left(1+\frac{\alpha^{2}}{n^{2}}\right)^{1 / 2} \approx 2
$$

Therefore, the right side of Formula (60) is as follows. 


$$
2 \pi \frac{n \hbar}{2}\left[1+\left(1+\frac{\alpha^{2}}{n^{2}}\right)^{1 / 2}\right] \approx 2 \pi n \hbar
$$

If the STR is taken into account, then it is evident that strictly speaking, Bohr's quantum condition does not hold, and it only holds approximately. That is,

$$
p_{\text {re }, n} \cdot 2 \pi r_{\text {re, } n} \approx 2 \pi n \hbar \text {. }
$$

\subsection{De Broglie's Hypothesis}

First, de Broglie's hypothesis (19) can be rewritten as follows.

$$
2 \pi r_{\mathrm{re}, n}=n \lambda_{n} \text {. }
$$

Here $\lambda_{n}$ is the wavelength of an electron with momentum $p_{\text {re, } n}$.

Using Formula (56), the left side of Formula (67) is,

$$
\begin{aligned}
2 \pi r_{\mathrm{re}, n} & =2 \pi \frac{r_{\mathrm{e}}}{2} \frac{\left(n^{2}+\alpha^{2}\right)^{1 / 2}}{\left(n^{2}+\alpha^{2}\right)^{1 / 2}-n}=2 \pi \frac{r_{\mathrm{e}}}{2} \frac{n^{2}+\alpha^{2}+n\left(n^{2}+\alpha^{2}\right)^{1 / 2}}{\alpha^{2}} \\
& =2 \pi \frac{n^{2} r_{\mathrm{e}}}{2 \alpha^{2}}\left[1+\frac{\alpha^{2}}{n^{2}}+\left(1+\frac{\alpha^{2}}{n^{2}}\right)^{1 / 2}\right] .
\end{aligned}
$$

Here,

$$
\frac{r_{\mathrm{e}}}{\alpha^{2}}=\frac{e^{2}}{4 \pi \varepsilon_{0} m_{\mathrm{e}} c^{2}}\left(\frac{4 \pi \varepsilon_{0} \hbar c}{e^{2}}\right)^{2}=4 \pi \varepsilon_{0} \frac{\hbar^{2}}{m_{\mathrm{e}} e^{2}}=a_{\mathrm{B}} .
$$

If the relationship in Formula (69) is incorporated into Formula (67),

$$
2 \pi r_{\mathrm{re}, n}=2 \pi \frac{n^{2} a_{\mathrm{B}}}{2}\left[1+\frac{\alpha^{2}}{n^{2}}+\left(1+\frac{\alpha^{2}}{n^{2}}\right)^{1 / 2}\right] \text {. }
$$

Next, let's consider the right side of Formula (67). Using the relationship of de Broglie, the right side of Formula (67) can be written as follows.

$$
n \lambda_{n}=n \frac{h}{p_{\mathrm{re}, n}} \text {. }
$$

If Formulas (24) and (52) are used here, Formula (71) becomes,

$$
n \lambda_{n}=n \frac{m_{\mathrm{e}} c \lambda_{\mathrm{C}}}{m_{\mathrm{e}} c}\left(\frac{n^{2}+\alpha^{2}}{\alpha^{2}}\right)^{1 / 2}=n^{2} \frac{\lambda_{\mathrm{C}}}{\alpha}\left(1+\frac{\alpha^{2}}{n^{2}}\right)^{1 / 2} .
$$

Here, we take into account the fact that the following relationship holds.

$$
\frac{2 \pi r_{\mathrm{e}}}{\alpha}=\lambda_{\mathrm{C}} .
$$

Next, if both sides of Formula (73) are divided by $\alpha$,

$$
\frac{2 \pi r_{\mathrm{e}}}{\alpha^{2}}=\frac{\lambda_{\mathrm{C}}}{\alpha}=2 \pi a_{\mathrm{B}} \text {. }
$$

If Formula (74) is used, then Formula (72) becomes as follows.

$$
n \lambda_{n}=2 \pi n^{2} a_{\mathrm{B}}\left(1+\frac{\alpha^{2}}{n^{2}}\right)^{1 / 2} .
$$


Next is a comparison of the right side of Formula (70) and the right side of Formula (75).

First, the part in parentheses on the right side of Formula (70) is regarded as approximately 2 . That is,

$$
1+\frac{\alpha^{2}}{n^{2}}+\left(1+\frac{\alpha^{2}}{n^{2}}\right)^{1 / 2} \approx 2
$$

Finally, $r_{\mathrm{re}, n}$ in Formula (70) is,

$$
r_{\mathrm{re}, n} \approx n^{2} a_{\mathrm{B}} .
$$

\section{Discussion}

1) First, if both sides of Formula (31) are squared, and multiplied by $m_{n}^{2} /\left(m_{\mathrm{e}}+m_{n}\right)$,

$$
\frac{m_{n}^{2}}{m_{\mathrm{e}}+m_{n}} \cdot \frac{v_{n}^{2}}{c^{2}}=\frac{\alpha^{2}}{n^{2}} \cdot \frac{m_{n}^{2}}{m_{\mathrm{e}}+m_{n}} .
$$

From this, the relativistic kinetic energy of the electron $K_{\mathrm{re}, n}$ is,

$$
K_{\mathrm{re}, n}=\frac{m_{n}^{2} v_{n}^{2}}{m_{\mathrm{e}}+m_{n}}=\frac{\alpha^{2} c^{2}}{n^{2}} \cdot \frac{m_{n}^{2}}{m_{\mathrm{e}}+m_{n}} .
$$

If the relationship in Formula (50) is used here,

$$
K_{\mathrm{re}, n}=\frac{\alpha^{2} c^{2}}{n^{2}}\left(\frac{n^{2}}{n^{2}+\alpha^{2}}\right) m_{\mathrm{e}}^{2} \cdot \frac{1}{m_{\mathrm{e}}\left[1+\left(\frac{n^{2}}{n^{2}+\alpha^{2}}\right)^{1 / 2}\right]} .
$$

Next, the following formula is multiplied with the numerator and denominator,

$$
1-\left(\frac{n^{2}}{n^{2}+\alpha^{2}}\right)^{1 / 2}
$$

When this is done,

$$
\begin{aligned}
K_{\mathrm{re}, n} & =\frac{\alpha^{2} m_{\mathrm{e}} c^{2}}{n^{2}}\left(\frac{n^{2}}{n^{2}+\alpha^{2}}\right)\left[1-\left(\frac{n^{2}}{n^{2}+\alpha^{2}}\right)^{1 / 2}\right]\left(1-\frac{n^{2}}{n^{2}+\alpha^{2}}\right)^{-1} \\
& =\frac{\alpha^{2} m_{\mathrm{e}} c^{2}}{n^{2}}\left(\frac{n^{2}}{n^{2}+\alpha^{2}}\right)\left[1-\left(\frac{n^{2}}{n^{2}+\alpha^{2}}\right)^{1 / 2}\right]\left(\frac{n^{2}+\alpha^{2}}{\alpha^{2}}\right) \\
& =m_{\mathrm{e}} c^{2}\left[1-\left(\frac{n^{2}}{n^{2}+\alpha^{2}}\right)^{1 / 2}\right] \\
& =m_{\mathrm{e}} c^{2}-m_{n} c^{2} .
\end{aligned}
$$

This enables derivation of Equation (45) from Equation (31).

Thus, the relativistic energy levels of the hydrogen atom are,

$$
E_{\mathrm{re}, n}=m_{n} c^{2}-m_{\mathrm{e}} c^{2}=m_{\mathrm{e}} c^{2}\left[\left(\frac{n^{2}}{n^{2}+\alpha^{2}}\right)^{1 / 2}-1\right] .
$$


Incidentally, the only quantum number included in Formula (82) is the principal quantum number $n$. Therefore, it is not the case that all the energy levels of the hydrogen atom can be derived from Formula (82). Also, with Bohr's quantum condition and de Broglie's hypothesis, it was possible to sketch a picture of the hydrogen atom, but with Formula (31), there is no picture of the electron. However, that is not a problem in quantum mechanics.

The relativistic energy levels of the hydrogen atom can be derived from Formula (31), and thus Formula (31) is a more substantial relationship than Bohr's quantum condition.

2) If the energy levels derived by Bohr (17c) are multiplied by the classical orbital radius (28),

$$
E_{\mathrm{BO}, n} r_{\mathrm{BO}, n}=-\frac{\alpha^{2} m_{\mathrm{e}} c^{2}}{2 n^{2}} \cdot \frac{r_{\mathrm{e}}}{\alpha^{2}} n^{2}=-\frac{m_{\mathrm{e}} c^{2}}{2} r_{\mathrm{e}} .
$$

Next, if we find the product of the relativistic energy levels derived in this paper (82) and the relativistic orbital radius (54),

$$
E_{\mathrm{re}, n} r_{\mathrm{re}, n}=\left(m_{n}-m_{\mathrm{e}}\right) c^{2} \cdot \frac{r_{\mathrm{e}}}{2} \frac{m_{\mathrm{e}}}{m_{\mathrm{e}}-m_{n}}=-\frac{r_{\mathrm{e}}}{2} m_{\mathrm{e}} c^{2} .
$$

The following relationship holds based on Formulas (83) and (84).

$$
\frac{E_{\mathrm{BO}, n} r_{\mathrm{BO}, n}}{E_{\mathrm{re}, n} r_{\mathrm{re}, n}}=1 .
$$

Also, the following equation holds due to Formula (10).

$$
E_{\mathrm{re}, n}=-\frac{1}{2} \frac{1}{4 \pi \varepsilon_{0}} \frac{e^{2}}{r_{\mathrm{re}, n}}=-\frac{1}{2} m_{\mathrm{e}} c^{2} \frac{r_{\mathrm{e}}}{r_{\mathrm{re}, n}} .
$$

This is because the following relationship holds due to Formula (86).

$$
E_{n} r_{n}=-\frac{1}{2} \frac{e^{2}}{4 \pi \varepsilon_{0}}=-\frac{1}{2} m_{\mathrm{e}} c^{2} r_{\mathrm{e}} .
$$

However, $E_{n} r_{n}$ does not signify a physical quantity. Therefore, Formula (87) does not have any special meaning. However, if $E_{n} r_{n}$ is always constant, then it can be used to verify this relationship. The product of Formulas (54) and (81c) derived in this paper is correct.

3) According to the discussion in this paper, the strict form of Bohr's quantum condition (23) is as follows.

$$
p_{\mathrm{re}, n} \cdot 2 \pi r_{\mathrm{re}, n}=2 \pi \frac{n \hbar}{2}\left[1+\left(1+\frac{\alpha^{2}}{n^{2}}\right)^{1 / 2}\right] .
$$

Also, strictly speaking, de Broglie's hypothesis becomes as follows.

$$
2 \pi r_{\mathrm{r}, n}=\frac{n \lambda_{n}}{2}\left[1+\left(1+\frac{\alpha^{2}}{n^{2}}\right)^{1 / 2}\right] .
$$

Thus, $r_{\mathrm{re}, n}$ is, 


$$
r_{\mathrm{re}, n}=\frac{1}{2 \pi} \frac{n \lambda_{n}}{2}\left[1+\left(1+\frac{\alpha^{2}}{n^{2}}\right)^{1 / 2}\right] .
$$

If this $r_{\mathrm{re}, n}$ is substituted for $r_{\mathrm{re}, n}$ in Formula (88), the following de Broglie relationship is obtained.

$$
\lambda_{n}=\frac{2 \pi \hbar}{p_{\text {re }, n}} .
$$

From this, it is evident that Formulas (88) and (89) are the same formula.

\section{Conclusions}

Quantum condition (23) was assumed first in Bohr's theory. Also, the energy levels of the hydrogen atom (17) and the orbital radius of the electron (22) were derived using that quantum condition. It was also possible to find the velocity of the electron from Formula (34).

However, this paper concludes that Bohr should have assumed the following formula to begin with

$$
\frac{v_{n}}{c}=\frac{\alpha}{n}
$$

From Formula (92) it is easy to find Formula (17). Formula (92) is built into the Bohr quantum condition. From this relationship, this paper can also determine, albeit partially, the relativistic energy levels of the hydrogen atom (82). However, Formula (82) cannot be found from Bohr's quantum condition.

On the other hand, the relativistic quantum condition (88) was inferred by substituting $p_{\mathrm{re}, n}$ or $r_{\mathrm{re}, n}$, derived by another method, into Bohr's quantum condition (21). Originally, $p_{\mathrm{re}, n}$ and $r_{\mathrm{re}, n}$ had to be derived in the reverse way from Formula (88).

Formula (88) is complicated, so it is difficult to assume in the beginning. Therefore, it is impossible to conclude that Formula (88) is the true quantum condition.

This paper concludes that Formula (92) is suitable as a new quantum condition to take the place of Bohr's quantum condition.

When deriving the energy levels of the hydrogen atom, Bohr should have assumed Formula (92).

\section{Acknowledgements}

In preparing the Introduction, the author referred to the writings in Japanese of Dr. H. Ezawa. Here I would like to express my gratitude to Dr. Ezawa. Also, I would like to express my thanks to the staff at ACN Translation Services for their translation assistance.

\section{Conflicts of Interest}

The author declares no conflicts of interest regarding the publication of this paper. 


\section{References}

[1] Bohr, N. (1913) On the Constitution of Atoms and Molecules. Philosophical Magazine, 26, 1-25. https://doi.org/10.1080/14786441308634955

[2] Suto, K. (2019) The Relationship Enfolded in Bohr's Quantum Condition and a Previously Unknow Formula for Kinetic Energy. Applied Physics Research, 11, 19-34. https://doi.org/10.5539/apr.v11n1p19

[3] Suto, K. (2015) An Unknown Physical Constant Missing from Physics. Applied Physics Research, 7, 68-79. https://doi.org/10.5539/apr.v7n5p68

[4] Sommerfeld, A. (1923) Atomic Structure and Spectral Lines. Methuen \& Co. Ltd, London, 528.

[5] Suto, K. (2011) An Energy-Momentum Relationship for a Bound Electron Inside a Hydrogen Atom. Physics Essays, 24, 301-307. https://doi.org/10.4006/1.3583810

[6] Suto, K. (2020) Theoretical Prediction of Negative Energy Specific to the Electron. Journal of Modern Physics, 11, 712-724. https://doi.org/10.4236/jmp.2020.115046 